This item was submitted to Loughborough's Research Repository by the author.

Items in Figshare are protected by copyright, with all rights reserved, unless otherwise indicated.

\title{
Perceptions of integrity in sport: insights into people's relationship with sport
}

PLEASE CITE THE PUBLISHED VERSION

https://doi.org/10.1080/19406940.2020.1747101

PUBLISHER

Informa UK Limited, trading as Taylor \& Francis Group

VERSION

AM (Accepted Manuscript)

PUBLISHER STATEMENT

This is an Accepted Manuscript of an article published by Taylor \& Francis in International Journal of Sport Policy and Politics on 4 May 2020, available online:

http://www.tandfonline.com/10.1080/19406940.2020.1747101.

\section{LICENCE}

CC BY-NC-ND 4.0

\section{REPOSITORY RECORD}

Manoli, Elisavet, Comille Bandura, and Paul Downward. 2020. "Perceptions of Integrity in Sport: Insights into People's Relationship with Sport”. Loughborough University. https://hdl.handle.net/2134/12248903.v1. 


\title{
Perceptions of integrity in sport; insights into people's relationship with sport
}

\begin{abstract}
Supported by a wealth of research, current UK Government policy emphasises the important role of sport in delivering social outcomes. What is not considered in the current policy and academia are the logically plausible adverse associations that would follow when sport is shown to lack integrity, an increasing phenomenon nowadays. If sport is found to lack integrity, then does this alter people's perceptions and their relationship with it? In this study, we aim to answer this question by examining people's views on the lack of integrity in sport and their propensity to participate, volunteer and spectate sport in the UK context. Data collected through 18 focus groups reveal a perceived 'normalisation' of the lack of integrity in sport and an overall belief that sport cannot manage its integrity. The findings suggest that paradoxically, even though sport is viewed as untrustworthy, people's propensity to participate, spectate and volunteer in sport remains unchanged. Consequently, even though sport is viewed as corrupt and unable to improve, its perceived role as a mechanism for social outcomes appears to remain unaffected, creating a potentially vicious circle in which sport has little to no pressing urgency or strong motivation to protect its integrity.
\end{abstract}

Keywords: Sport integrity, sport corruption, sport participation, sport spectatorship, volunteering, focus groups. 


\section{Introduction}

It is widely argued that sport plays an important role in creating the cultures and identities of our communities (Houlihan and Malcolm, 2015), reducing inequalities (Collins and Kay, 2003) and developing social capital (Elmose-Østerlund and van der Roest, 2017; Kumar, Manoli, Hodgkinson and Downward, 2018). Indeed, there is a plethora of research advocating for sport as a mechanism for social and community development. For example, engagement, participation, voluntary involvement and spectatorship have been found to foster social inclusion and cohesion (Coleman, 1988; Arai and Pedlar, 2003; Lockstone-Binney, Holmes, Smith and Baum, 2010; Collins and Heere, 2018). As a result it is argued that individuals within a community will collaborate for their mutual benefit and that of society to behave with integrity and have high moral attitudes (Hooghe and Stolle, 2003). This belief is a fundamental reason why UK Government policy makers and key stakeholders invest in and support sport systems that are believed to deliver social or community outcomes through participation, volunteering and spectatorship (Schulenkorf, Sherry, and Rowe, 2016). What these policy makers seem to disregard or neglect is the existence of a rather darker side of sport linked with negative social outcomes, such as aggression and exclusion (Numerato and Baglioni, 2012), which is nevertheless ignored over the above mentioned positive outcomes of sport. While the dark side of sport does not detract from its link with positive social outcomes, overlooking it could be considered another symptom of what Coakley calls the 'Great Sport Myth' (2015, p. 402). The Great Sport Myth refers to the wider belief that sport can be a universal mechanism for social good, guided by its wider inherent purity and goodness, which in turn precludes critical discussions and shapes, often inaccurately, our overall understanding of sport's role in society (Coakley, 2015; 2017; Pielke, 2016).

Indeed, the existence of the dark side of sport can be further emphasised by the lack of integrity in sport, which although not a new phenomenon, is increasingly becoming the 
new epidemic in sport world-wide (Maennig 2005). Instances of lack of integrity range from individual athlete transgressions (e.g. doping in cycling, running, etc - Solberg, Hanstad and Thøring, 2010), to club scandals (e.g. match-fixing in Greece, Italy, Turkey, etc - Manoli and Antonopoulos, 2015; Yilmaz, Manoli and Antonopoulos, 2019) and federation-wide corruption (e.g. bribing in the IOC and FIFA - Jennings, 2011), in both commercialised, professional and elite sports (such as the examples mentioned above), and in noncommercialised, amateur sports (e.g. chess - Solberg and Olderøien, 2018; Zaksaite, 2013) and grassroots sports (e.g. grassroots football - Nowy and Breuer, 2017), which raises concerns about the integrity of sport in general (McNamee, 2013).

In this exploratory study, we aim to examine people's views on the lack of integrity in sport and their perceptions on how it might affect their engagement with sport. The article is structured as follows: firstly, the theoretical foundations of integrity in sport are explored. Secondly, the methodology and limitations of our research are presented, along with opportunities for future research. Finally, the findings of the research are then presented and discussed, followed by a discussion of the implications of this study.

\section{Integrity in sport}

Integrity, as Gardiner, Parry and Robinson (2017, p. 6) argue 'is a significantly undertheorised and under-conceptualised value within sports'. Despite its widespread use by sport federations and policy organisations (e.g., IOC, FIFA, Transparency International, Play the Game, INTERPOL, Council of Europe), integrity in sport has yet to be clearly defined and theorised, with recent work on the topic underling the need for further attention (Cleret, McNamee and Page, 2015; Vanden Auweele, Cook and Parry, 2016). A recent study by the European Union further highlighted this lack of a universal definition of integrity in sport, by collating a plethora of definitions and arguing that despite the increased interest the topic has 
received within both academia and policy, additional research is needed in order for our understanding of the issue to be furthered (European Commission, ECORYS and Manoli, 2019).

Most discussions around integrity issues include words like honesty, trust, values, ethics, morals, beliefs, respect and fairness, which Erhard and Jensen (2017) argue can influence both how a system is designed and functions, and how people within it behave. As such, they argue that an out-of-integrity behaviour by individuals or an out-of-integrity function of a system makes them incoherent and inconsistent with their intended role and purpose, damaging in this way their value. Geeraert (2016) argues that this out-of-integrity behaviour results in 'ethical scandals' that are increasingly appearing in sport due to its commercialisation, its instrumentalisation (or use) by politics, and the organisational characteristics of the sport system. As a result, it becomes a fertile ground for instances of lack of integrity, which cannot be challenged only by better governance, but also need to be met with tighter regulation (Chappelet, 2018).

Currently, integrity in sport can be used to describe honesty, ethics and sport related values such as equality on the playing field, while often it is presented as the opposite to corruption in sport (Haberfeld and Sheehan, 2013). Corruption in sport is also a rather underresearched and under-conceptualised area, as studies on topic suggest. Maenning (2005) argues that corruption in sport is mostly evident on athletes' performance and their choice to refrain from achieving a win, and on sporting officials who conduct themselves in ways contradicting with the values of sport organisations, sport in general, and society overall. Gorse and Chadwick (2011) suggest that corruption in sport includes any activity that is illegal, immoral or unethical, that is aiming at changing the result of a sport competition for one's gain. Finally, Masters (2015, p. 133) introduces one of the most cited definitions of corruption in sport, according to which it is: "the deviation from public expectations that 
sport will be played and administered in an honest manner" While this definition captures a broader view of what corruption in sport can be perceived as, while emphasising the element of integrity, it is largely based on the belief that there exists an inherent integrity that is associated with or 'expected' in sport.

Archer (2016) argues that integrity in sport has yet to be fully conceptualised, offering a re-examination of the conceptualisation of the term in his study. He argues that integrity in sport can be broadly viewed in three ways: as coherence of one's self (strength of will), as practical identity (one's character) or as a social view (in response to social norms). At the same time, he suggests that integrity should be described as a virtue rooted in three different sites: the personal (of the individuals involved in sport), the institutional (of the organisations involved in sport) and within sport themselves. Following Archer's study, Gardiner et al. (2017) suggest that four aspects of integrity in sport exist. First, the personal integrity of the individuals involved in sport under any capacity (e.g. athlete, administrator, coach, referee). These individuals are expected to maintain their personal integrity through their actions and thus protect their identity, values and reputation. When a contractual agreement exists between an individual and sport (e.g. professional athlete, sport administrator) then personal integrity is also closely connected to professional integrity. Second, organisational integrity, which is similar to personal integrity, assumes that organisations strive to represent their values and identity and thus sustain their reputation. MacIntyre (1981) and McNamee (2008) argue that maintaining organisational integrity ensures that sport organisations adhere to the social practices that they are supposed to protect and promote, by focusing primarily on the moral principles that sport promotes and expects. Third, procedural integrity in sport events, which is also based on the personal integrity discussed above. In this case, since sport is a social practice, procedural integrity in a sport event would ensure that individuals involved in a sport contest would maintain their personal integrity and thus promote and protect the 
values of the sporting competition, making the event trustworthy (Gardiner et al., 2017). And forth, the often debated inherent integrity of sport which implies that sport participants are entitled to moral equality (Simons, 1999). This moral equality is linked with honesty, conscientiousness, ethics and values that are believed to be coherent with the axioms, or fundamental truths and perceptions, of the origins of sport (Becker, 1998; Chatziefstathiou, 2011), while often being contested as a glorified but less accurate representation of sport's values nowadays (Coakley, 2015; 2017).

At the same time, studies on corruption in sport have focused on the lack of integrity in sport. These include a focus on: the lack of personal integrity, for example when examining cases of doping and anti-social behaviour (Boardley and Kavussanu, 2007; Kirkwood, 2012); the lack of organisational and procedural integrity, when incidents of financial irregularities and match-fixing are analysed (Manoli and Antonopoulos, 2015; Manoli, Antonopoulos and Levi, 2016), and even debating the original idea of the inherent integrity of sport (McNamee, 2013; Kihl, 2017). These studies have argued that sport integrity and often all four of its aspects can be absent from modern sport worldwide. Interestingly, despite commercial and elite sports and corruption within them often capturing media's attention, corruption in sport is believed to be a wide-spread phenomenon, extending to non-commercialised, amateur and grassroots sport as well. In fact, recent studies in amateur and grassroots sports underline the proliferation of corrupt practices within them (Nowy and Breuer, 2017). Numerato (2016) argues that corruption in less commercialised sport settings is frequently taken for granted, acting as a public secret which is widely known but not acted upon. Numerato furthers his argument by suggesting that sport settings in general allow for corruptive behaviours to be tolerated and for secrecy around them to be preserved. Numerato's (2016) and Manoli, Antonopoulos and Bairner's (2017) studies question the existing integrity within sport overall, raising a wider doubt on whether the four 
aspects of integrity in sport and in particular the inherited integrity of sport are still applicable in today's sporting context.

These studies raise a wider question on how sport is perceived in today's society bearing the lack of sport integrity in mind. If sport is found to lack integrity, then does this alter people's perceptions and their relationship with it? In other words, in this study we aim to explore people's views on the lack of integrity in sport and their propensity to participate, volunteer and spectate sport in the UK context.

\section{Methodology}

Since the aim of this exploratory study was to gain an in-depth understanding of people's perceptions, focus groups were selected as the ideal data collection method, which would allow us to obtain rich and insightful data from selected groups of individuals rather than from a statistically representative sample of a broader population (O Nyumba, Wilson, Derrick and Mukherjee, 2018). Focus groups were used in this study not as a multiple interview tool, but as a way of exploring people's opinions through their discussion with their peers, as Cyr (2016) suggests. While focus groups have been criticised for their dynamics, including social pressures, groupthink and desirability bias, it is argued that the pressures and biases they entail are similar to the ones induced by everyday conversations (Hollander, 2004; Gamson, 1992), and thus focus groups can be a useful tool in exploring people's understanding and interpretation of a phenomenon.

Mindful of social desirability effects and potential bias existing within focus groups, efforts were made for homogenous groups to be developed, allowing for in-depth discussions among the participants (Smithson, 2000). While biases within each group cannot be avoided, Kidd and Parshall (2000) argue that in a homogenous group a discussion through agreement and disagreement among the participants can lead to a more accurate account of the their views. 
Efforts were also made from the researchers to ensure that not only the collective voice of each focus group was heard, but also each individual opinion as expressed, with follow up questions asked in order to ensure that all participants' views were noted. Emphasis was drawn on any points of disagreement within each focus group, in order to ensure that all viewpoints were discussed equally, as Kidd and Parshall (2000) suggest. Attention was also paid to ensure that all members in each focus group were participating in the discussions, by probing any silent participants to agree or disagree with previous statements made (Smithson and Díaz, 1996). Additionally, debates were encouraged in each focus group, allowing for individual views to be expressed and multiple perspectives on each topic to be elicited, rather than a jointly produced position, in an attempt to limit the groupthink effect, which is nonetheless a limitation in the use of focus groups as it is discussed below (Cyr, 2016).

Throughout these focus groups, discussions among each group were facilitated by the researchers to allow participants' views and perceptions to emerge through the conversation, rather than through investigative questions (Smithson, 2000). However, a list of key questions was designed (see Appendix A) to facilitate the conversation in the focus groups, with the researchers adopting the role of moderator in each focus groups, allowing the discussion to continue and develop freely and uninterrupted among the participants. The questions used were tested in a pilot focus group.

A number of sampling techniques were used in order for the participants to be recruited for this study. Firstly, convenience sampling was adopted by recruiting individuals who resided in the UK, who were available and willing to participate (approached through email, telephone and social media outputs). This sampling method was chosen due to its ease and efficiency in allowing us to recruit participants in the beginning of the study, bearing in mind the relatively large target number of participants for the research (Atkinson and Flint, 2001). Secondly, snowball sampling was used by asking individuals who had already 
participated to recommend others. This method enabled us to develop homogenous groups that would encourage in-depth discussions among the participants and potentially minimise the bias and desirability effects within them (Smithson, 2000). Finally, selective sampling was adopted through calls on social media in order to ensure that diversity existed in the participants of this study, in terms of both demographic characteristics and interest and involvement in sport. This method allowed us to respond to possible limitations arising from the above two sampling techniques, such as the lack of diversity in participants and possible bias (Atkinson and Flint, 2001), and thus particular efforts were made in order for participants with characteristics that were missing from the ones already included in the study to be recruited through selective sampling. The two moderators conducting the focus groups developed a saturation grid, a data analysis tool that lists the major topics arising from the data, which was updated following each focus group. Through the regular update of the grid, data saturation was monitored and discussed between the researchers (Fusch and Ness, 2015). Through their discussion and realisation that no new codes or themes were emerging from the final two focus groups, the researchers were able to identify that saturation of data had occurred.

Eighteen focus groups were conducted for this study between February and May 2018 reaching data saturation. The average duration of the focus groups was 75 minutes and with an average number of five to six participants per focus group. Consequently, there were 99 participants across the 18 focus groups. The demographic characteristics of the participants can be found in Appendix B. As it can be seen in the appendix, participants' interest or engagement with sport was not a criterion in their recruitment to this exploratory study, allowing us insights into more diverse viewpoints regarding integrity in sport.

All focus groups were recorded and transcribed as soon as possible after each focus group was over, producing an average of 10 single spaced pages per group. The focus group 
transcripts were subsequently sent to a proportion of the participants $(10 \%$ of the overall participants in this study), so that any inaccuracies or misinterpretations could be pinpointed and for the authenticity of the focus groups to be verified, while assisting in limiting any potential bias from the researchers (Kidd and Parshall, 2000).

The data was analysed manually following Braun and Clarke's (2006, p.16) six phases of thematic analysis (see Appendix C for key themes and illustrative quotes), which allowed for the codes identified in text to be grouped under overarching themes and subthemes. Coding the data manually was a laborious and lengthy process that involved examining the transcriptions meticulously while colour coding the codes and themes that emerged (different shades of colour for each theme) and annotating the transcripts. Designing and updating the saturation grid throughout the data collection process assisted in developing the initial codes, which were then applied in the first two transcripts independently by each member of the team, before comparing the points of agreement and disagreement among them. Through the cross-examination of the codes developed, the coders exchanged ideas that assisted in designing the thematic template of the study that was used in the remaining transcripts. Regular meetings to update the template took place when the need arose, in which the intercoder reliability was also calculated (on average 90\%). Intracoding was also implemented, by coding the same transcripts at different points in time, with intracoding reliability scoring above $96 \%$ every time. This careful intercoder and intracoder process, as well as the regular meetings among the researchers allowed for their individual biases to be controlled, while increasing the confidence of the coding process (Boyatzis, 1998). The final 'thematic-map' was then designed with all themes, sub-themes and codes that emerged, allowing for all themes to be reviewed and refined collectively by the researchers.

Before the findings of the study are discussed, its limitations need to be acknowledged. Efforts were made for multiple sampling strategies to be employed in order 
for a representative sample of participants to be included, while the focus groups and the data analysis was conducted by multiple researchers closely following a carefully designed strategy in order to limit potential bias and increase the robustness of the study. Nonetheless, due to the nature of the study and the data collection and analysis methods selected, participant and researcher biases should be acknowledged. Similarly, and despite the moderators' efforts to avoid it, groupthink should be acknowledged as a potential limitation of this study, arising from the focus group methodology adopted. Moreover, due to the nature and context of this study, as well as the focus group data collected, the transferability of the findings may be limited. Nevertheless, through this exploratory study, we do not aim to generalise the findings, but instead offer insights to people's views on the lack of integrity in sport in the UK context, which can act as an indication of similar patterns of perceptions to be observed in similar cases. Future research could explore this topic on a wider scale and in similar or less similar settings, while a longitudinal study could also examine the effects of the lack of integrity on both the perceptions and the behaviour of individuals in terms of participation, spectatorship and volunteering.

\section{Findings}

\section{The 'normalisation' of lack of integrity in sport}

The discussions made it evident that all participants were aware of several incidents of lack of integrity in both national and international sport, with their views indicating that a lack of integrity in sport is perceived to be common knowledge. In fact, it could be argued that their statements indicated an acceptance towards the overall phenomenon of lack of integrity that was evident throughout all focus groups conducted as the following quotes illustrate. 
It's nothing I've not heard before. It's been plastered all over the news for the past year so it's not a shock to me... It's so big, so deep rooted, so high profile like we have seen it many times so you know things like this have to come out. (Male, 27, focus group 7)

Corruption in sport, is now the reality of sport, right? I mean I can't think of any sport that hasn't had a bad story come out in the last few years. In a way, there's nothing that anyone could ever say about corruption in sport that would shock me anymore. (Male, 24, focus group 5)

As both quotes suggest, this awareness and almost acceptance of the lack of integrity was due to the number of incidents that have been presented and discussed often and in detail in both national and international media in recent years. Throughout all focus groups the participants were able and eager to discuss a number of incidents, ranging from individual transgressions to federation-wide scandals affecting both the sporting and the management side of sport.

Remember swimming, gymnastics, snooker? It was on the news last night. There's something about corruption in sport. It's now the reality of it, right? (Male, 36, focus group 10)

The examples discussed included incidents of various perceived severity stretching from small scale instances of wrong doings, to large scale crimes and offenses, as the quote below illustrates. 
Commercial, non-commercial, big, small, it doesn't really matter. It's everywhere in sport. On the top of my head I can think of so many examples that have happened only in the past few years. And they're pretty much everywhere, from the Olympics to the local league where my son plays some Sundays. (Male, 35, Focus group 11)

The argument illustrated through this quote highlights a key point that found all participants in agreement; that the lack of integrity in sport is perceived to be a reality in all aspects of sport. The researchers often probed the participants to elaborate further on the points raised, in order for any differences to be expressed in regard to the perceived severity of corruption or the level of commercialisation or competitiveness in each sport, as well as for any disagreements within the focus groups to be noted. The responses given by the participants, however, highlighted that this universal perception of a 'normalised' lack of integrity in sport did not differentiate between diverse levels of commercialisation or competitiveness, as the following quote shows.

Let's be honest here, we've all heard about this. I mean, you can't really avoid it. It's on the news, on the papers, on the internet, everywhere. From the big FIFA scandal or the Olympic doping one, to the smaller local leagues where players get picked because they know the manager, or the poor referee who has to pick sides before making a decision. (Female, 37, focus group 13)

Throughout all focus groups the inherent integrity of sport was questioned repeatedly and was instead replaced with the notion that morality and ethics in sport are in reality assumed to be absent, and instead a lack of integrity is almost accepted as an integral part of sport nowadays. In fact, as one participant argued, people have now become 'numb' to it due to the 
volume of incidents of lack of integrity in sport and the resistance or inability of sport to combat it. Once again, a distinction was not made among different sports, levels of commercialisation or popularity, with all sports deemed equally unable to manage their integrity.

\section{Sport cannot manage its integrity}

The discussion on the lack of integrity in sport, was closely followed by a debate on whether sport can or cannot manage its integrity. Throughout all focus groups, there was strong acceptance that sport is unable or even incompetent to manage and preserve its integrity, as the quote below shows.

But what we read is still happening now in 2018. It's obviously been going on for so many years. It's still happening now. Why isn't anything in place to stop it? Are you telling me they can't put anything in place to stop that? I think they just don't want to. (Female, 38, focus group 4)

I remember reading about stories like that for years now and yet nothing seems to change. It might be actually getting worse. For all I know in a few years' time, it's going to be even worse than today...I don't know what they're doing, but I don't think they're doing something to fight it. And if they are, it's clearly not working, right? (Female, 41, focus group 12)

The argument that sport needs to improve in order to protect its integrity was made by all participants, who were also suggesting that any actions currently taken are unsuccessful or insufficient, as the quotes above also illustrate. When the discussion touched upon measures 
taken against corruption in sport, it is worth underlining that not all participants were aware of the efforts made, while the consensus was that since corruption in sport persists, then the actions taken are inadequate.

While a number of potential reasons about the insufficient response to corruption were mentioned, such as the lack of formal structures for control and accountability within the sporting system, the majority of the participants argued that it is 'the overall culture of sport' that is to be blamed.

I don't think they're managing it. I don't think they're doing enough. If they were, we wouldn't be reading about it on the papers all the time. And in all honestly, I don't know who is to blame. It might be difficult to change and you might need time and resources, but in all fairness, I don't think sport truly wants to change. Sport has its own peculiar, private culture that lets it exist as it is. (Male, 32, focus group 7)

The perceived culture was described as rather secretive, exclusive or even elitist, aiming primarily at self-preservation. As such, it was argued that when an issue would appear within sport, it would be deemed easier to supress it and ostracise those who disagree, rather than fix the problem in hand. This in turn would mean that any individuals who would react to an incident of lack of integrity would have to first assess whether reporting the incident would have any repercussions on themselves before acting upon it. Discussion on this perceived culture of sport emerged in all focus groups, with participants often having strong views on how deeply rooted it is in sport nowadays and how much it has influenced sport's integrity or lack thereof. According to the participants, if sport will not fight to protect its integrity and on the contrary might choose to ostracise them, then the primary factor that affects one's 
decisions is their own personal involvement in sport, either in terms of their career or their enjoyment in both professional and non-professional sport.

But that's what sport is today, right? This idea of a rather secretive society where it's so difficult to be an insider. So, once you're in and you see all these things, would you talk? Because if you do, you might be kicked out of it and I don't know if anyone is willing to risk it. So my friend who accepted to pay for her son to play, if she said no and reported the guy, would they stop it, or would her son be kicked out of the team? That's why we think of sport as corrupt, because no one within it will do anything about it. (Female, 30, focus group 13)

According to the participants, this has in turn created a rather peculiar dilemma for the people involved in sport; if reporting or reacting to the lack of integrity does not guarantee a response, and provided that there are no systems for individuals to come forward in a way that would protect their anonymity, then is responding to the lack of integrity more important than sport for them. This would consequently suggest that the personal integrity of the individuals involved, as well as their professional integrity when they have a contractual agreement with sport, might be compromised due to the pressures exerted by this culture of sport, as participants' examples illustrated.

I mean, is there a mechanism to protect anyone who talks? If I volunteer in the local rugby club and I see something there, would they let me keep helping or would they kick me out? If volunteering is more important for me, then I might choose to turn the blind eye and keep doing my thing. Now what if I volunteered in my son's rugby club. 
If speaking up meant that they'd kick him out of practice, then I would definitely not do it. (Female, 28, focus group 2)

Following researchers' questions on how universal this perceived culture of sport might be, participants were able to offer examples, as the above quote illustrates, that were not only limited to individuals employed within the sport industry who might risk losing their jobs if they do not succumb to this culture. They also included cases of amateur and leisure sport where individuals would risk themselves or their families being ostracised by their local club as participants, spectators or volunteers if the perceived norm was not upheld. As participants argued, it is due to this perceived culture that sport has not been able to protect its integrity, while the perpetuation of this phenomenon might have actually allowed for the problem to consolidate and even escalate over the years, creating the impression that sport is not willing or able to manage its integrity, as the following quote show.

It's never really going to change. It's been highlighted. People have been made more aware of it, almost accepting it, rather than having a strategy to change it... We all know there is something in sport that is not quite right. (Female, 50, focus group 15)

While the commercialisation of sport was often mentioned as an important influencing factor in today's sporting landscape, examples of lack of integrity in a number of sports were mentioned by the participants, ranging from heavily commercialised to niche and grassroots sports. In fact, when asked about their views on whether lack of integrity is a phenomenon encountered mainly in commercial or highly competitive levels of sport, all participants argued that even though more instances of corruption are bound to be found in more commercial or competitive sports, lack of integrity is believed to be a wide-spread occurrence 
in every level and aspect of sport, from the competition to the management side, as it was mentioned above. Similarly, reacting or opposing to an incident of lack of integrity would equally not take place in both commercial and non-commercial sport, according to the participants, since the perceived culture of concealment and secrecy and the dilemma it poses on individuals, as it was discussed above, is believed to be a common occurrence in all sports. As such, the lack of integrity in sport was presented by the participants as a standard, common practice that is accepted and to be expected nowadays.

\section{Sport cannot be trusted}

Following the belief that sport lacks integrity and is also unable to manage it, there was a consensus among the participants that sport cannot be trusted. Participants emphasised that the lack of transparency and accountability encountered in sport, particularly in the way in which sport is governed, had a ripple effect in the way in which they felt not only about organised sport, but also sport in general. As it was discussed above, the participants argued that the perceived secretive culture of sport perpetuates this lack of trust they were feeling, as the following quotes suggest.

I think there are corrupted people in sport at most levels. You can't trust people anywhere to be honest. I don't trust FIFA because of what's come out in the papers. I don't trust coaches at the youngsters' level. I think it's really bad at the minute. (Male, 24, focus group 3)

I don't and I can't trust sport overall. I don't follow sport, but there's always something on the news about corruption in sport which makes me not trust it at all. If it was one thing, we'd be able to move past it I guess, but we've been hearing about 
corruption for too long and in any possible aspect of sport, so it's just too much to pretend it's not happening and in one way or the other you end up not trusting sport at all. (Female, 32, focus group 16)

As the quotes show, this distrust towards sport found all participants in agreement, despite their varying levels of interest and involvement in sport. Interestingly, as the second quote illustrates, even the participants who did not express an interest towards sport suggested a distrust towards it due to the proliferation of incidents of corruption in sport, broadcasted often through the media. Notably, even though most participants used examples of lack of integrity in organised commercial and competitive sports to justify their mistrust toward sport, their statements did not seem to limit their feelings of mistrust to these particular sports, suggesting a potential spill over effect to their perceptions of other sports as well. As a result, when participants were probed to elaborate more on their distrust towards sport no exceptions were offered, since as one participant suggested, any sport that appears to have integrity can prove corrupted if one looks close enough.

\section{Engagement with sport remains unaffected}

Despite the proclaimed distrust towards sport, engagement with sport was deemed too important to be affected by the lack of integrity, with participants arguing that their interest and propensity to spectate, participate and volunteer in sport remained unchanged, as the following quote shows.

If something like this came up in hockey, I'd still play it. My whole family would still play it. I've been brought up with it and I love it. It's my own love for the sport that would overrule everything. (Female, 25, focus group 5) 
Similar views were expressed by all participants, including those who did not suggest such an emotional attachment to a sport, or those that did not identify as fans of sport overall, since 'this sort of thing happens everywhere really'.

I don't really play sport, so that doesn't affect me, but I am not sure it would affect me if I was playing anyway. Or if I was watching sport or doing anything around it. I mean people, society overall is corrupt, so sport is just a part of society, right? (Male, 35 , focus group 11)

The argument expressed above suggests the existence of moral disengagement among the participants. As such, lack of integrity is perceived and presented as a common and widespread phenomenon nowadays, and thus sport is believed to be just another context in which the phenomenon manifests itself. The discussions within the focus groups illustrated that the lack of integrity was viewed as a separate phenomenon and as such a clear distinction was made between the phenomenon and the context, allowing individuals the moral disengagement to justify their continuous support and interest in sport.

I'd take part and I'd encourage my son to get involved in sport if he has an interest. I'd encourage him for the social side, making friends and the challenges it brings. It certainly wouldn't put me off from encouraging him into sport. (Male, 32, focus group 1) 
If Tour de France were passing from out here, I'd go there and my friends would definitely come with me. We'd have a good time and I don't think we'd even think that the athletes were doped. We'd still have fun. (Female, 24, focus group 12)

As the quotes suggest, the positive social outcomes of sport engagement were still referenced as the reason behind the lack of change in participants' interest and propensity to participate, spectate and volunteer in sport, despite their reported lack of trust and discontent with sport's inability to manage its integrity. As a result, the same participants who argued that sport cannot be trusted appeared to be willing to participate, spectate and volunteer in sport, as well as encourage their families and friends to do so, even with sports where examples of lack of integrity were offered. Despite the perceived wide-spread lack of integrity and sport's inability to manage it, no change in their interest or propensity to engage and be involved in sport was reported by any of the participants. Sport, even when corrupt and not trusted, was presented as a mechanism for social outcomes and thus as an indispensable part of participants' lives who argued that their interest and intent to be involved as a participant, volunteer or spectator would not diminish.

Even when such an interest in sport was not expressed, participants argued that any hypothetical willingness to engage in sport would not diminish due to the lack of integrity, since sport was viewed once again as another context in which lack of integrity occurs. As a result, it was argued that any potential social outcomes emerging through this hypothetical engagement with sport would still exist, despite their lack of trust toward sport, and thus engagement with sport would not be considered as endorsement or encouragement to its lack of integrity. 
If I felt like playing a sport or joining a club or volunteering for an event, I'd do it to socialise and make friends. And I would be ok to do it, even while knowing that sport in general and the sport I'd choose to play or help in particular was corrupt. That's because I'd still make friends and be part of the team and that's what sport is about. I mean I still have a bank account even though I know that all banks are corrupt.

(Male, 28, focus group 14)

This quote illustrates the moral disengagement discussed above, in which the lack of integrity within sport is acknowledged, but the interest and willingness to engage with sport remains unchanged due to its positive outcomes for the individuals involved. Sport is therefore viewed as an important, maybe too important, vehicle to deliver social outcomes irrespectively of the profoundly reported lack of trust towards it and its inability to manage its integrity. As such, individuals are therefore put in the position of balancing the costs and benefits of being involved in sport and, as the quotes above show, view their personal experiences and aspirations, as well as the expected benefits of the engagement with sport, as being more important than the lack of integrity within sport.

\section{Discussion and Implications}

While the prevalence of lack of integrity in sport that academics have argued about (Maenning, 2005; Kihl, 2017; Manoli et al., 2017) was mirrored in the participants' views, the findings of our study suggested that the perceived insufficient or unsuccessful response this has been met with in sport has normalised the phenomenon in people's minds, something that sport administrators and policy makers need to take into account. The perceived severity of incidents of lack of integrity does not seem to affect or alter these perceptions towards sport, with all white, grey and dark incidents (Heidenheimer, 2002), taking place in all 
competitive, elite, commercial, amateur or grassroots sport, resulting in the same response of expectancy and acceptance according to participants. While sport is a complex ecosystem consisting of various forms and sectors, ranging from organised, competitive and elite, to grassroots, amateur and self-organised (Manoli, 2014), participants' perceptions of the lack of integrity in sport did not seem to distinguish among them, resulting in a wider feeling of mistrust toward sport. As such the lack of integrity was presented as an integral part of sport, contradicting the earlier belief of its inherent integrity (Simons, 1999; Gardiner et al., 2017). This can in turn raise an important question on the applicability of the definition of corruption itself. If the definition of corruption in sport suggests that corruption is "the deviation from public expectations that sport will be played and administered in an honest manner" (Masters, 2015, p. 113), and yet the public expectation of sport is one tainted with increased incidents of corruption and lack of integrity, then the basis of the definition might not be applicable anymore, potentially suggesting the need for an updated definition of sport corruption. Public expectations, according to the participants of this study, are not those of equality, morality and fairness in sport, mirroring the values that sport was said to represent historically (Becker, 1998; Chatziefstathiou, 2011). Instead they appear to have been replaced by those of acceptance and numbness to the lack of integrity, shaking the basis of the belief that sport should be perceived as an intrinsically social good and echoing the criticism expressed by Coakley $(2015 ; 2017)$. If the public expectation is that corruption is now an accepted and anticipated part of sport, then one could even question whether the deviation from public expectations in no longer corruption, but instead integrity in sport.

If this is the case and sport is a system in which corruption is perceived to be expected, as the findings of this study suggest, then implications arise for both sport administrators and policy makers alike. For the latter, questions can be raised on whether and to what extent public policy, such as the one by the UK Government, can or should support 
and encourage sport engagement through participation, volunteering and spectatorship. If sport is indeed no longer viewed as a social good, then public policy supporting and promoting it could be reviewed to better account for the updated people's perceptions of its nature and the lack of integrity within it. For the former, people's perceptions of sport as they emerge through this study, should influence and potentially alarm sport administrators' practice and future strategy. Since the lack of integrity in sport is perceived to be expected, and yet sport's response is viewed as inadequate or insufficient, then future strategy should account for it, by incorporating urgent action plans to better manage sport's integrity and tackle manifestations of corruption within it. Additionally, since this study's findings highlight the need for additional communication and transparency on behalf of sport, sport administrators should ensure that their actions to protect and promote sport's integrity, as well as their potential effectiveness, are widely communicated to the media and public, while ensuring that a positive light is also shed on the overall sport system in order to improve people's perceptions.

According to the participants, sport is currently perceived as untrustworthy and is instead viewed as fraudulent, corrupt and unable or unwilling to improve, with a presumed culture of concealment and secrecy that perpetuates the problem and often compromises the often expected personal integrity of the individuals involved in sport (McNamee, 2013; Archer, 2016; Gardiner et al., 2017). Paradoxically, however, and despite the mistrust expressed by the participants, their conviction that sport can deliver social outcomes through engagement appears to be unaffected. In spite of the severe criticism expressed towards sport, participants indicated that their interest and willingness to participate, spectate or volunteer in sport remains entirely unchanged due to the expected results that their engagement with sport entails, with the perceived severity of incidents of lack of integrity having similarly no impact. As a result, and despite participants' emphasised mistrust towards it, sport's ability to 
deliver social outcomes that is cited in academia and Government policy appears to be considered possible (HM Government, 2015; Schulenkorf et al., 2016; Collins and Heere, 2018). Nevertheless, since this possibility was examined in this study through participants' intended future engagement with sport, additional research is needed in order for the actual future engagement with sport through participation, volunteering and spectatorship to be examined, allowing us a better view on the possibility of sport to still deliver social outcomes unaffected, despite the emphasised mistrust towards it.

The reason why the paradox mentioned above might exist within sport could be justified by the moral disengagement expressed by the participants (Boardley and Kavussanu, 2007; Kirkwood, 2012), according to which the lack of integrity in sport mirrors the lack of integrity in our society overall. As a result, sport is merely another context in which corruption exists, which paired with its highly valued expected social outcomes, gives individuals the moral licensing needed to consider sport important despite their lack of trust and criticism on its ability to manage its integrity (Klotz and Bolino, 2013).

From a policy and management perspective, however, if the lack of integrity in sport does not affect the interest and willingness to participate, spectate and volunteer in sport, then sport has little to no pressing urgency or strong motivation to challenge it. In other words, a potentially vicious circle is created in which sport is not trusted and is believed to be unable or unwilling to improve its practices regarding managing or protecting its integrity. Yet at the same time, since this lack of integrity appears to not be paired with a decreased propensity to participate, spectate and volunteer, the same individuals that do not trust sport give it little incentive to improve, perpetuating in this way the existence and proliferation of lack of integrity in sport, something that sport administrators and policy makers should take into consideration. While the future results of this lack of trust toward sport are difficult to be predicted without additional research, by people maintaining the same levels of participation, 
spectatorship and volunteering, the lack of integrity in sport does no other harm than to sport itself. 


\section{References}

Arai, S. and Pedlar, A. (2003) Moving beyond individualism in leisure theory: a critical analysis of concepts of community and social engagement, Leisure Studies, 22(3), 185-202

Archer, A. (2016). On sporting integrity. Sport, Ethics and Philosophy, 10(2), 117-131.

Atkinson, R. and Flint, J. (2001) Accessing hidden and hard-to-reach populations: Snowball research strategies. Social Research Update, 33(1), 1-4.

Becker, T. E. (1998) Integrity in organizations: Beyond honesty and conscientiousness. Academy of Management Review, 23(1), 154-161.

Boardley, I. D. and Kavussanu, M. (2007) Development and validation of the moral disengagement in sport scale. Journal of Sport and Exercise Psychology, 29(5), 608-628.

Boyatzis, R. E. (1998) Transforming qualitative information: Thematic analysis and code development. London: Sage.

Braun, V. and Clarke, V. (2006) Using thematic analysis in psychology. Qualitative research in psychology, 3(2), 77-101.

Chappelet, J. L. (2018). Beyond governance: The need to improve the regulation of international sport. Sport in Society, 21(5), 724-734.

Chatziefstathiou, D. (2011) Changes and continuities of the ideology of Olympism in the modern Olympic Movement. Sport in Society, 14(3): 332-344.

Cleret, L., McNamee, M. and Page, S. (2015) 'Sports integrity' needs sports ethics (and sports philosophers and sports ethicists too) Sport, Ethics and Philosophy, 9(1), 1-5.

Coakley, J. (2017). Sport in society: Issues and controversies. $12^{\text {th }}$ edition. New York: McGraw Hill Education. 
Coakley, J. (2015). Assessing the sociology of sport: On cultural sensibilities and the great sport myth. International Review for the Sociology of Sport, 50(4-5), 402-406.

Coleman, J. (1988) Social capital in the creation of human capital. American Journal of Sociology, 94, 95-120.

Collins, M. and Kay, T. (2003) Sport and Social Exclusion, London: Routledge

Collins, D. R. and Heere, B. (2018) Sunday afternoon social capital: an ethnographic study of the Southern City Jets Club. European Sport Management Quarterly, 18(4), 439-458.

Cyr, J. (2016) The pitfalls and promise of focus groups as a data collection method. Sociological Methods \& Research, 45(2), 231-259.

Elmose-Østerlund, K. and Van der Roest, J. W. (2017) Understanding social capital in sports clubs: participation, duration and social trust. European Journal for Sport and Society, 14(4), 366-386.

Erhard, W. and Jensen, M. C. (2017) Putting Integrity Into Finance: A Purely Positive Approach, Capitalism and Society, 12(1), 1-91.

European Commission, ECORYS and Manoli, A. E. (2019) European Union Report: Mapping of corruption in sport in the EU. Brussels: European Commission.

Fusch, P. I. and Ness, L. R. (2015) Are we there yet? Data saturation in qualitative research. The qualitative report, 20(9), 1408-1416.

Gamson, W. A. (1992) Talking politics. Cambridge: Cambridge University Press.

Gardiner, S., Parry, J. and Robinson, S. (2017) Integrity and the corruption debate in sport: where is the integrity?. European sport management quarterly, 17(1), 6-23. 
Geeraert, A. (2016) Corruption and governance in sport. In U. Wagner, R. K. Storm \& K.

Nielsen, When sport meets business: Capabilities, challenges, critiques, London: Sage.

Haberfeld, M.R. and Sheehan, D. (Eds.) (2013) Match-fixing in international sports: Existing processes, law enforcement, and prevention strategies. New York: Springer.

Heidenheimer, A. J. (2002) Perspectives on the Perception of Corruption, edited by: A.J. Heidenheimer and M. Johnston. Political corruption: Concepts and contexts, 141-154. London: Transaction Publishers.

Her Majesty’s Government. (2015) Sporting future: A new strategy for an active nation. London: Cabinet Office.

Hollander, J. A. (2004) The social contexts of focus groups. Journal of contemporary ethnography, 33(5), 602-637.

Hooghe, M. and Stolle, D. (2003) Introduction: Generating social capital. In M. Hooghe \& D. Stolle(Eds.), Generating social capital: civil society and institutions in comparative perspective (pp. 1-18). Basingstoke: Palgrave Macmillan.

Houlihan, B. and Malcolm, D. (Eds.) (2015) Sport and society: a student introduction. London: Sage.

Jennings, A. (2011) Investigating corruption in corporate sport: The IOC and FIFA. International Review for the Sociology of Sport, 46(4), 387-398.

Klotz, A. C. and Bolino, M. C. (2013) Citizenship and counterproductive work behavior: A moral licensing view. Academy of Management Review, 38(2), 292-306.

Kidd, P. S., \& Parshall, M. B. (2000) Getting the focus and the group: enhancing analytical rigor in focus group research. Qualitative health research, 10(3), 293-308. 
Kihl, L. A. (2017) Corruption in Sport. London: Routledge.

Kirkwood, K. (2012) Defensive doping: Is there a moral justification for “if you can't beat ‘em—Join 'em?”. Journal of Sport and Social Issues, 36(2), 223-228.

Kumar, H., Manoli, A.E., Hodgkinson, I.R. and Downward, P. (2018) Sport participation: From policy, through facilities, to users' health, well-being, and social capital. Sport Management Review, 21(5), 549-562.

Lockstone-Binney L., Holmes L., Smith, K. \& Baum, T. (2010) Volunteers and volunteering in leisure: Social science perspectives. Leisure Studies, 29(4), 435-455.

MacIntyre, A. (1981) After virtue. London: Duckworth.

Maennig, W. (2005) Corruption in international sports and sport management: Forms, tendencies, extent and countermeasures. European Sport Management Quartely, 5(2), 187225.

Manoli, A. E. (2014) The football industry through traditional management analysis.

In Scandinavian Sport Studies Forum, 5(1), 93-109.

Manoli, A. E. and Antonopoulos, G. A. (2015) 'The only game in town?': football matchfixing in Greece. Trends in Organized Crime, 18(3), 196-211.

Manoli, A. E., Antonopoulos, G. A. and Bairner, A. (2017) The inevitability of corruption in Greek football. Soccer \& Society, 1-17.

Manoli, A. E., Antonopoulos, G. A. and Levi, M. (2016) Football clubs and financial crimes in Greece. Journal of Financial Crime, 23(3), 559-573.

McNamee, M. (2008) Morality play: Sports, virtues and vices. London: Routledge. 
McNamee, M. (2013) The integrity of sport: Unregulated gambling, match fixing and corruption. Sport, Ethics and Philosophy, 7(2), 173-174.

Masters, A. (2015) Corruption in Sport: From the playing field to the field of policy. Policy and Society, 34(2), 111-123.

Nowy, T. and Breuer, C. (2017) Match-fixing in European grassroots football. European Sport Management Quarterly, 17(1), 24-44.

Numerato, D. (2016) Corruption and public secrecy: An ethnography of football matchfixing. Current Sociology, 64(5), 699-717.

Numerato, D. and Baglioni, S. (2012) The dark side of social capital: An ethnography of sport governance. International Review for the Sociology of Sport, 47(5), 594-611.

O Nyumba, T., Wilson, K., Derrick, C.J. and Mukherjee, N. (2018) The use of focus group discussion methodology: Insights from two decades of application in conservation. Methods in Ecology and Evolution, 9(1), 20-32.

Pielke, R. (2016) Obstacles to accountability in international sports governance. Transparency International, global corruption report: Soccer. Oxon: Routledge. Schulenkorf, N., Sherry, E. and Rowe, K. (2016) Sport for development: An integrated literature review. Journal of sport management, 30(1), 22-39.

Simons, T.L. (1999) Behavioral integrity as a critical ingredient for transformational leadership. Journal of Organizational Change Management, 12(2), 89-104.

Smithson, J. (2000) Using and analysing focus groups: limitations and possibilities. International journal of social research methodology, 3(2), 103-119. 
Smithson, J. and Díaz, F. (1996) Arguing for a collective voice: Collaborative strategies in problem-oriented conversation. Text-Interdisciplinary Journal for the Study of Discourse, 16(2), 251-268.

Solberg, H.A., Hanstad, D.V. and Thøring, T. A. (2010) Doping in elite sport-do the fans care? Public opinion on the consequences of doping scandals. International Journal of Sports Marketing and Sponsorship, 11(3), 2-16.

Solberg, H. A. and Olderøien, L. (2018) The Challenges of Hosting Major Events Owned by International Federations: A Case Study of the 2014 Chess Olympics. Event Management, 22(4), 595-608.

Vanden Auweele, Y., Cook, E. and Parry, J. (Eds.) (2016) Ethics and governance in sport: The future of sport imagined. London: Routledge.

Yilmaz, S., Manoli, A.E. and Antonopoulos, G.A. (2019) An anatomy of Turkish football match-fixing. Trends in Organized Crime, 22(4), 375-393.

Zaksaite, S. (2013) Match-fixing: the shifting interplay between tactics, disciplinary offence and crime. The International Sports Law Journal, 13(3-4), 287-293. 